
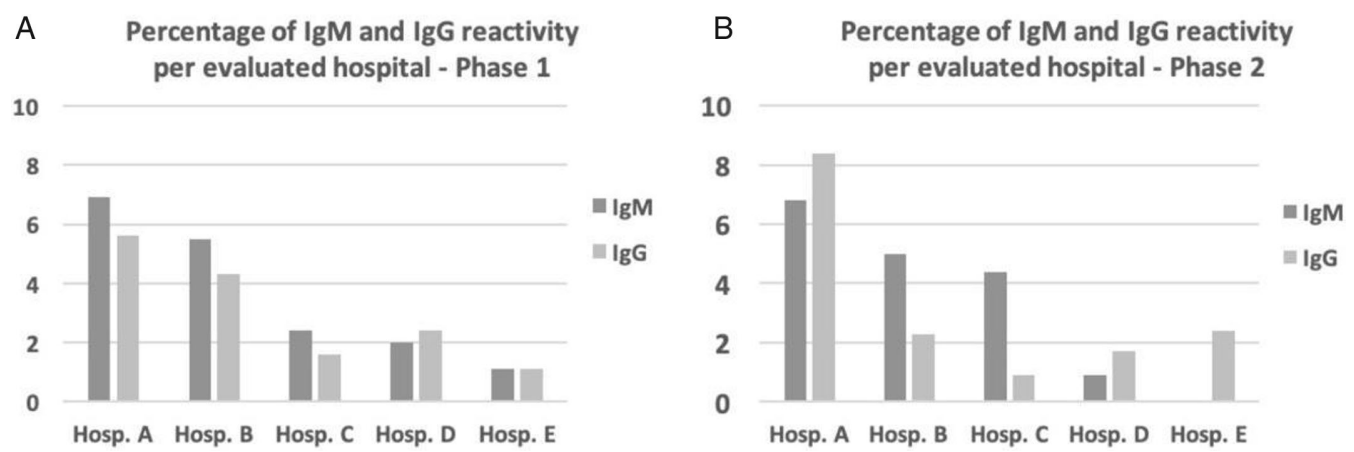

Fig. 1. Positivity for COVID-19 IgM and IgG antibodies in the first (A) and second (B) phases of the study, in the 5 hospitals studied. Hospitals are not identified in this slide, they are randomly named A-E.
Financial support. This study was sponsored by Instituto Cultural Floresta. We are in debt with Claudio Goldsztein and Marcelo Wallauer for supporting this study, in addition to all donors to Instituto Cultural Floresta.

Conflicts of interest. All the authors declare no conflicts of interest related to this article.

\section{References}

1. Lan FY, Lan FY, Filler R, et al. COVID-19 symptoms predictive of healthcare workers SARS-CoV-2 PCR results. PLoS One 2020;15(6):e0235460.

2. Wong SCY, Kwong RT, Wu TC, et al. Risk of nosocomial transmission of coronavirus disease 2019: an experience in a general ward setting in Hong Kong. J Hosp Infect 2020;105:119-127.

3. Bergh MFQK- Van Den. SARS-CoV-2 infection in 86 healthcare workers in two Dutch hospitals in March. medRxiv 2020. doi: 10.1101/2020.03.23. 20041913.

4. Sotgiu G, Barassi A, Miozzo M, et al. SARS-CoV-2 specific serological pattern in healthcare workers of an Italian COVID-19 forefront hospital. BMC Pulm Med 2020;20:203.

5. Moscola J, Sembajwe G, Jarrett M, et al. Prevalence of SARS-CoV-2 antibodies in healthcare personnel in the New York City area. JAMA 2020;324: 893-895.

6. Malfertheiner S, Brandstetter S, Roth S, et al. Immune response to SARS$\mathrm{CoV}-2$ in healthcare workers following a COVID- 19 outbreak: a prospective longitudinal study. J Clin Virol 2020;130:104575.

7. Long QX, Tang XJ, Shi QL, et al. Clinical and immunological assessment of asymptomatic SARS-CoV-2 infections. Nat Med 2020;26:1200-1204.

8. Liu A, Wang W, Zhao X, et al. Disappearance of antibodies to SARSCoV-2 in a COVID-19 patient after recovery. Clin Microbiol Infect 2020; 26:1703-1705.

9. Paiva KJ, Grisson RD, Chan PA, et al. Validation and performance comparison of three SARS-CoV-2 antibody assays. J Med Virol 2021;93:916-923.

\title{
Vaccines in the coronavirus disease 2019 (COVID-19) era: Game theory applications
}

\author{
Marios Papadakis MD, MBA, $\mathrm{PhD}^{1}$ (1) and Nikolaos Spernovasilis MD, MPH \\ ${ }^{1}$ University Witten-Herdecke, Germany and ${ }^{2}$ School of Medicine, University of Crete, Heraklion, Greece
}

To the Editor-Vaccines are among the greatest inventions of science, preventing millions of deaths worldwide annually. Currently, the lack of a coronavirus disease 2019 (COVID-19) vaccine has led to

Author for correspondence: Marios Papadakis, E-mail: marios_papadakis@yahoo.gr Cite this article: Papadakis M and Spernovasilis N. (2022). Vaccines in the coronavirus disease 2019 (COVID-19) era: Game theory applications. Infection Control \& Hospital Epidemiology, 43: 957-958, https://doi.org/10.1017/ice.2021.125 a pandemic that has brought the whole world to a near standstill. Although international collaborations to guarantee equitable access have been established, as COVID-19 vaccines are approved, national egotism is expected. ${ }^{1}$ Game theory is the science of strategy and interactive decision making, where the outcome depends not only on one's actions but also on the actions of others. ${ }^{2}$ Herein, we present applications of game theory in vaccine allocation. 
The well-known Prisoner's Dilemma is the most common game theory paradigm. It proves that 2 individuals who act in their own self-interest do not produce the optimal outcome. As countries try to secure vaccine availability for their citizens, they may fail to slow the spread of the virus elsewhere, which harms human society overall. For example, if Germany concentrates on making the vaccine available only for all German citizens while the outbreak grows in Belgium, Switzerland, and other neighboring countries, a shortage of ICU beds could result in these countries. However, such a shortage led to critical care patients being transferred to Germany, where the ICU capacity was sufficient.

The Tragedy of the Commons refers to bad outcomes for the whole system, when individual self-interests conflict with the common good. It is based on a 200 -year-old concept that originally described a group of shepherds who let their sheep graze on a shared field, resulting in the grass getting eaten down to the roots and, thus, all sheep dying. As more and more "wealthy" countries order large numbers of vaccines for their citizens, concerns arise that, if the manufacturing capacity limit is reached, low-income countries may have to wait $2-3$ years to get vaccines. The COVAX initiative aims to prevent such vaccine hoarding by guaranteeing vaccines to $20 \%$ of each country's most vulnerable population. Such an approach gives shepherds (in this case the pharmaindustry) time to plant new grass (in this case more vaccines) for the sheep (in this case the population). Moreover, approval of $>1$ effective vaccine means more grass for a given number of individuals.

The Free Rider Dilemma refers to an individual or a group who uses a resource or receives a benefit without having any contribution to it. In the COVID-19 era, free riders can be considered all persons that benefit from herd immunity without exposing themselves to the vaccine. At the country level, free riders can be considered countries that have not participated in the negotiations for the vaccine distribution (eg, countries that will receive vaccines just because they are EU members) or developing countries that do not participate in the COVAX facility but take advantage of its decisions.

In the Volunteer's Dilemma, he who goes first loses, but if no one tries all lose. For example, University of Oxford/AstraZeneca (Oxford/AZ) partnership was the first to conclude a vaccine agreement with the European Commission. ${ }^{3}$ Oxford/AZ promised vaccine at uniform price worldwide $(\$ 2.90)$ and was granted protection from future product liability claims. The Pfizer/ BioNTech vaccine was approved for use in the United Kingdom, and although it is priced at $\$ 20.00$, the UK government also granted the manufacturer protection from being sued. ${ }^{4}$ This example may also indicate reciprocity - a situation in which, after adopting a cooperative strategy on the first move, the player proceeds by copying the moves of the other players.

Finally, the Stag Hunt Dilemma describes a conflict between safety and cooperation. According to the scenario, a group of hunters go hunting. If all hunters work together and kill the stag, it will provide shared meat for all. If they work at their immediate self-interest, they may succeed killing a hare (which is worth less than a stag), providing adequate meat only for the individual. The problem is that there is no guarantee for a stag, so the hunter's dilemma when he sees a hare is whether to kill it or to sacrifice his immediate self-interest for the risk of not eating anything. In the COVID-19 era, the stag is a highly effective vaccine, which, at the beginning, could not be guaranteed. Oxford/AZ reported an overall vaccine efficacy of $70 \%$. To increase this efficacy, Oxford/AZ decided to work with the makers of Russia's Sputnik-V vaccine to test a combined shot, based on 1 of 2 vectors of Sputnik-V. This could produce greater and longer-lasting immunity.

In conclusion, human cooperation is the only driving force that can minimize such dilemmas and provide a fair, uniform, and equitable COVID-19 vaccine allocation.

\section{Acknowledgments.}

Financial support. No financial support was provided relevant to this article.

Conflicts of interest. All authors report no conflicts of interest relevant to this article.

\section{References}

1. Greer SL. National, European, and global solidarity: COVID-19. Eurohealth 2020;26:104-108.

2. McFadden DW, Tsai M, Kadry B, Souba WW. Game theory: applications for surgeons and the operating room environment. Surgery 2012;152:915-922.

3. Ng WH, Liu X, Mahalingam S. Development of vaccines for SARS-CoV-2. F1000Res 2020. doi: 10.12688/f1000research.25998.1.

4. Hafner M, Yerushalmi E, Fays C, Dufresne E, Van Stolk C. COVID-19 and the Cost of Vaccine Nationalism. Santa Monica, CA: RAND Corporation; 2020.

\title{
Why comparing coronavirus disease 2019 (COVID-19) and seasonal influenza fatality rates is like comparing apples to pears
}

\author{
Marios Papadakis MD, MBA, PhD (10 \\ University Witten-Herdecke, Witten, Germany
}

To the Editor-The coronavirus disease 2019 (COVID-19) pandemic is a once-in-a-lifetime event for humanity. By the end of March 2021, 130 million cases had been confirmed worldwide and $>2.8$ million people have died, with a case fatality rate (CFR)

Author for correspondence: Marios Papadakis, E-mail: marios_papadakis@yahoo.gr Cite this article: Papadakis M. (2022). Why comparing coronavirus disease 2019 (COVID-19) and seasonal influenza fatality rates is like comparing apples to pears. Infection Control \& Hospital Epidemiology, 43: 958-959, https://doi.org/10.1017/ice.2021.140 of nearly $2.2 \%$. At the beginning, the fact that COVID-19 typically presents as a flu-like illness, led many healthcare professionals and scientists to adopt strategies traditionally used to fight seasonal influenza because both entities seemed to have similar patterns of viral shedding. This resemblance quickly resulted in direct comparisons of these separate entities in terms of fatality rates as well.

Although COVID-19 and seasonal influenza share several common clinical and epidemiological characteristics, a one-to-one comparison of fatality rates is not reliable. In fact, such 